\title{
Commentary Frontal EEG for intensive care unit sedation: treating numbers or patients? \\ Peter V Sackey
}

Department of Anesthesiology, Surgical Services and Intensive Care Medicine, Karolinska University Hospital Solna, 17176 Stockholm, Sweden

Corresponding author: Peter V Sackey, peter.sackey@karolinska.se

See related research by Haenggi et al., http://ccforum.com/content/12/5/R119

Published: 23 October 2008

This article is online at http://ccforum.com/content/12/5/186

(c) 2008 BioMed Central Ltd

Critical Care 2008, 12:186 (doi:10.1186/cc7029)

\begin{abstract}
In this issue of Critical Care, Dr Haenggi and co-workers present a study evaluating bispectral index (BIS), state entropy (SE) and response entropy in 44 patients sedated in the intensive care unit (ICU). As in recent studies attempting to correlate frontal electroencephalogram (EEG) measurements with clinical evaluations of sedative efficacy, there is considerable overlap in numerical EEG values and different clinical levels of sedation. This precludes the use of these monitors for monitoring or titrating sedation in the critically ill. Despite many attempts, no study has yet presented data showing improved outcome with the use of EEG monitors in ICU sedation. Meanwhile, clinical sedation protocols have emerged, improving important endpoints in critically ill patients needing sedation. A major underlying problem in applying EEG monitors in the ICU is that they have been developed for measuring anesthetic depth and the related risk of recall, rather than the acknowledged endpoints of sedation, namely reduction of anxiety and discomfort. Until an 'objective' monitor is developed to measure the degree of such symptoms, physicians should continue treating patients and not numbers.
\end{abstract}

In this issue of Critical Care, Dr Haenggi and co-workers from Bern University Hospital present a study evaluating bispectral index (BIS), state entropy (SE) and response entropy (RE) during sedation in 44 patients in the intensive care unit (ICU) [1]. The authors used a meticulous protocol with parallel SE, RE and BIS measurements, web camera monitoring and a computer program for noting sedative administration and interventions - to find a clinical indication for the use of entropy or BIS during ICU Ramsay Sedation Scale-targeted sedation.

Statistically significant but poor correlation was found between the electroencephalogram (EEG) measurements and clinical assessment; correlation coefficients for RE and SE were -0.372 and -0.360 , respectively. For BIS and clinical assessment the correlation coefficient was -0.426 . These results, together with an overlap of entropy and BIS values between clinical sedation levels, led the authors to conclude that "BISIndex or Entropy do not add information which can be used to guide sedation in the general ICU population."

Other investigators have attempted to find correlation between EEG measurements of sedation and clinical parameters, similarly finding considerable overlap of displayed values between clinically different sedation levels and also close correlation with facial electromyographic activity [2-4]. The conclusions of these studies are identical to those of $\mathrm{Dr}$ Haenggi and co-workers, namely that BIS $[2,3]$ and entropy [4] are not reliable in describing the level of sedation or guiding sedative administration in ICU patients.

After more than a decade of use, the role of EEG parameters for sedation monitoring in the ICU is yet to be defined. For BIS - probably the most studied EEG parameter for ICU sedation - no study has yet shown clear benefit when used for sedation monitoring of ICU patients [5]. In contrast, new methods for clinical monitoring and titration of sedatives and analgesics have led to significant improvements in patient outcomes [6-8]. A daily wake-up test during sedation and mechanical ventilation has been shown to reduce time for mechanical ventilation and may also reduce ICU and hospital length of stay [7] and reduce complications of critical illness [9]. In a recent study, combining daily awakenings with spontaneous breathing tests additionally improved one-year survival, the effect probably depending on a lesser likelihood of oversedation [8]. Modifying sedation regimens may also modify patients memory panorama from the ICU and longterm psychological status $[10,11]$.

It may be tempting to introduce plug-and-play EEG monitors for measuring anesthetic depth - such as BIS - in the ICU.

$\mathrm{BIS}=$ bispectral index $; \mathrm{EEG}=$ electroencephalogram; $\mathrm{ICU}=$ intensive care unit; $\mathrm{RE}=$ response entropy; $\mathrm{SE}=$ state entropy. 
The concept of a number on a monitor informing clinicians how to titrate drug doses for adequate sedation is appealing. The problem with applying BIS for ICU sedation is that it was not originally designed to detect the main indications for sedative administration, namely discomfort and anxiety [12]. The anesthesia end-point that BIS primarily was developed to target and is marketed for [13] - amnesia - may not be a valid end-point for ICU sedation. Amnesia from the ICU is not necessarily a good thing [14]. Furthermore, given the data from the study and previous studies of BIS in ICU sedation, aiming for a target BIS-interval in ICU patients would likely lead to clinical problems of under- or oversedation in individual patients [1-4]. Acknowledged endpoints of ICU sedation are rather optimal patient comfort and safety [12]. EEG measures may not reflect the efficacy of sedation or analgesia with regard to these endpoints.

The previously prevailing idea of sleep being necessary for comfort in ICU patients may have emanated from transferring the anesthesia endpoint - hypnosis - from the operating room into the ICU, at a time when mechanical ventilation was less refined than it is today. New ventilators with more patientfriendly modes of ventilation may cause less patient-ventilator dysynchrony during light to moderate sedation, thereby potentially reducing sedative requirements. The development of new analgosedation concepts highlight this quandary of old and new sedation end-points in ICU patients $[15,16]$.

While EEG monitoring in patients receiving neuromuscular blocking treatment may be of some value given the lack of other methods for monitoring sedation depth [5], there is presently no evidence or rationale for the use of BIS or entropy in monitoring or titrating sedation of ICU patients. A number on a monitor indicating 'adequate sedation' can not replace the common sense of sedating a patient with clinical signs of discomfort or agitation, or reducing sedation in a unresponsive patient, unless patient outcome studies prove such an approach to be successful. The reasonable current sedation end-point should not, therefore, be deep sedation, a certain BIS or entropy value, but rather patient comfort and safety. Before considering new monitors for ICU sedation, we need to be confident that they make a difference in these end-points.

For now, let's treat patients and not numbers.

\section{Competing interests}

The author declares that they have no competing interests.

\section{References:}

1. Haenggi M, Ypparila-Wolters H, Bieri C, Steiner C, Takala J, Korhonen I, Jakob SM: Entropy and bispectral index for the assessment of sedation, analgesia and the effects of unpleasant stimuli in critically ill patients: an observational study. Critical Care 2008, 12:R119.

2. Sackey PV, Radell PJ, Granath F, Martling CR: Bispectral Index ${ }^{\mathrm{TM}}$ as a predictor of sedation depth during isoflurane or midazolam sedation. Anaesth Intensive Care 2007, 35:348-356.
3. Nasraway SA Jr, Wu EC, Kelleher RM, Yasuda CM, Donelly AM: How reliable is the Bispectral index in critically ill patients? A prospective, comparative, single-blinded observer study. Crit Care Med 2002, 30:1483-1487.

4. Walsh TS, Ramsay P, Lapinlampi TP, Särkelä MO, Viertiö-Oja HE, Meriläinen PT: An assessment of the validity of spectral entropy as a measure of sedation state in mechanically ventilated critically ill patients. Int Care Med 2008, 34:308-314.

5. LeBlanc JM, Dasta JF, Kane-Gill SL: Role of the Bispectral Index in sedation monitoring in the ICU. Ann Pharmacother 2006, 40:490-500.

6. Brook AD, Ahrens TS, Schaiff R, Prentice D, Sherman G Shannon W, Kollef MH: Effect of a nursing-implemented sedation protocol on the duration of mechanical ventilation. Crit Care Med 1999, 27:2609-2615.

7. Kress JP, Pohlman AS, O'Connor MF, Hall JB: Daily interruption of sedative infusions in critically ill patients undergoing mechanical ventilation. N Engl J Med 2000, 342:1471-1477.

8. Girard TD, Kress JP, Fuchs BD, Thomason JW, Schweickert WD, Pun BT, Taichman DB, Dunn JG, Pohlman AS, Kinniry PA, Jackson JC, Canonico AE, Light RW, Shintani AK, Thompson JL, Gordon SM, Hall JB, Dittus RS, Bernard GR, Ely EW: Efficacy and safety of a paired sedation and ventilator weaning protocol for mechanically ventilated patients in intensive care (Awakening and Breathing Controlled trial): a randomised controlled trial. Lancet 2008, 371:126-134.

9. Schweikert WD, Gehlback BK, Pohlman AS, Hall JB, Kress JP: Daily interruption of sedative infusions and complications of critical illness in mechanically ventilated patients. Crit Care Med 2004, 32:1272-1276.

10. Sackey PV, Carlswärd C, Martling C-R, Sundin Ö, Radell PJ: Short- and long-term follow-up of ICU patients after sedation with isoflurane and midazolam - a pilot study. Crit Care Med 2008, 36:801-806.

11. Kress JP, Gehlback B, Lacy M, Pliskin N, Pohlman AS, Hall JB: The long-term psychological effects of daily sedative interruption on critically ill patients. Am J Respit Crit Care Med 2003, 168:1457-1461.

12. Jacobi J, Fraser GL, Coursin DB, Riker RR, Fontaine D, Wittbrodt ET, Chalfin DB, Masica MF, Bjerke HS, Coplin WM, Crippen DW, Fuchs BD, Kelleher RM, Marik PE, Nasraway SA Jr, Murray MJ, Peruzzi WT, Lumb PD: Clinical practice guidelines for the sustained use of sedatives and analgesics in the critically ill adult. Crit Care Med 2002, 30:119-141.

13. Aspect Medical Systems: BIS monitoring and awareness [http://www.aspectmedical.com/professionals/safety/]

14. Jones C, Griffiths RD, Humphris G, Skirrow PM: Memory, delusions, and the development of acute posttraumatic stress disorder-related symptoms after intensive care. Crit Care Med 2001, 29:573-580.

15. Ruokonen E, Parviainen I, Jakob SM, Nunes S, Kaukonen M Shepherd ST, Sarapohja ST, Bratty JR, Takala J; for the Dexmedetomidine for Continuous Sedation Investigators: Dexmedetomidine versus $\mathrm{propofol} / \mathrm{midazolam}$ for long-term sedation during mechanical ventilation. Int Care Med 2008, [E-pub ahead of print].

16. Park G, Lane M, Rogers S, Bassett P: A comparison of hypnotic and analgesic based sedation in a general intensive care unit. Br J Anaesth 2007, 98:76-82. 